\title{
Singular stress fields at corners in flip-chip packages
}

\author{
Nanshu Lu, Zhen Zhang ${ }^{\mathrm{a}}$, Juil Yoon ${ }^{\mathrm{b}}$, and Zhigang Suo ${ }^{\mathrm{c}}$ \\ School of Engineering and Applied Sciences, Harvard University, Cambridge, MA 02138, USA
}

\begin{abstract}
An electronic device integrates diverse materials, and inevitably contains sharp features, such as interfaces and corners. When the device is subject to thermal and mechanical loads, the corners develop intense stress and are vulnerable sites to initiate failure. This paper analyzes stress fields at corners in flip-chip packages. The stress at a corner is a linear superposition of two modes of singular fields, with one mode being more singular than the other. The amplitudes of the two modes are represented by two stress intensity factors of dissimilar dimensions. To determine the stress intensity factors, we analyze the flip-chip structures under two loading conditions: stretching of the substrate and bending of the substrate. We show that the less singular mode may prevail over the more singular mode for some stretching-bending combinations. The relative significance of the two modes of stress fields also varies with materials, and with the substrate to chip thickness ratio.
\end{abstract}

Keywords: flip-chip package, corners, split singularities, stress intensity factors

a) Current address: Microsoft Corp., Silicon Valley Campus, 1065 La Avenida, Mountain View, CA 94043

b) Current address: Department of Mechanical Systems Engineering, Hansung University, Seoul 136-792, South Korea.

c) Corresponding author

Email: suo@seas.harvard.edu

Postal address: 29 Oxford St. Pierce Hall 309, Cambridge, MA 02138

Phone: 617-495-3789 Fax: 617-496-0601 


\section{Introduction}

In a flip-chip package, a silicon chip is flipped, with the face containing active devices connected to a substrate by solder bumps and underfill. The silicon chip is about $1 \mathrm{~cm}$ wide and $0.7 \mathrm{~mm}$ thick. The substrate has a width of several centimetres and a thickness of several millimetres. The material of the substrate can be either a ceramic or an organic. For example, in the mid-1990s ceramic substrates were replaced by organic laminates like FR4 (Flame Retardant 4) or BT (Bismaleimide and Triazine) resin. These organic substrates have a lower dielectric constant, have smaller thermal mismatch with the mother board, and are easier to process metallization. [1] Nevertheless, the organic substrates have several disadvantages, such as adsorption of moisture, large thermal mismatch with the silicon chip, severe warpage during assembly. [2, 3] As a result, novel ceramic substrates have also been developed; for example, Low Temperature Co-fired Ceramic (LTCC), a ceramic-glass composite with elastic and thermal properties comparable to those of silicon, is a potential choice for the substrate. LTCC is fired at sufficiently low temperature so that copper can be used in the metallization. The ceramic substrate also offers superior hermetic and mechanical stability to protect the chip. [4] Table 1 lists the properties of silicon and the two materials for the substrate.

A structure like a flip-chip package contains diverse materials and sharp features such as interfaces and corners. When the structure is subject to thermal and mechanical loads, the stress field intensifies at the corners, making them vulnerable sites to initiate failure. [3, 5-13] A challenge to analyze stress in flip-chip packages is the large variation in length scales, from the chip-substrate level of several centimetres down to the smallest transistors of several nanometres. To fully capture the chip-substrate behaviour, a multi-scale finite element method, known as global-local submodeling, is widely adopted. [9, 11, 14] Simulations of this kind, however, are 
tedious and expensive. Improved understandings of significant features in such structures are valuable.

This paper focuses on chip-substrate corners shown in Fig. 1(a). As discussed in the next section, we will apply the concept of split singularities to analyze the stress field at the corners. While the mechanics of split singularities at corners has been studied, here we apply the approach to an engineering structure to elucidate the relative significance of the two modes of singular stress fields. The paper is organized as follows. Section 2 summarizes the concept of split singularities. Section 3 shows how to calculate the two stress intensity factors and the mode angle under combined stretching and bending of the substrate. In Section 4, we investigate the relative contributions of the two singular modes for two substrate materials: FR4 and LTCC. Section 5 studies the significance of each mode for various chip-substrate geometries.

\section{Split singularities}

For a linearly elastic bimaterial with traction prescribed on the boundary, Dundurs [15] demonstrated that the stress field depends on elastic constants through two dimensionless parameters:

$$
\begin{gathered}
\alpha=\frac{E_{c}\left(1-v_{s}^{2}\right)-E_{s}\left(1-v_{c}^{2}\right)}{E_{c}\left(1-v_{s}^{2}\right)+E_{s}\left(1-v_{c}^{2}\right)} \\
\beta=\frac{1}{2} \frac{E_{c}\left(1+v_{s}\right)\left(1-2 v_{s}\right)-E_{s}\left(1+v_{c}\right)\left(1-2 v_{c}\right)}{E_{c}\left(1+v_{s}\right)\left(1-2 v_{s}\right)+E_{s}\left(1+v_{c}\right)\left(1-2 v_{c}\right)}
\end{gathered}
$$

where $E$ is Young's modulus, and $v$ Poisson's ratio. The subscripts “c” and "s” refer to the chip and the substrate, respectively. By requiring $E>0$ and $0 \leq v \leq 0.5$, the Dundurs parameters are

confined within a parallelogram in the $(\alpha, \beta)$ plane, with vertices at $(1,0),(1,0.5),(-1,0)$ and $(-1,-0.5)$ 
Figure 1(b) magnifies the bimaterial corner. A system of polar coordinates $(r, \theta)$ is attached, with the origin coinciding with the intersection of the edge of the chip and the surface of the substrate. The two materials are bonded along the interface $\theta=0^{\circ}$. The chip occupies the quarter space, $0^{\circ} \leq \theta \leq 90^{\circ}$, and the substrate occupies the half space, $-180^{\circ} \leq \theta \leq 0^{\circ}$. Both the chip and the substrate are taken to be linearly elastic and isotropic. All edges and interfaces are assumed to be perfectly sharp.

We next summarize the main results of the singular stress fields around the corner. [1619] Each component of the stress takes the form of $\sigma_{\mathrm{ij}} \sim \mathrm{r}^{-\lambda}$, where $\lambda$ is the exponent of the singular field. This singular stress field is determined by an eigenvalue problem. [16-23] The exponent is commonly restricted in the interval $0<\operatorname{Re}(\lambda)<1$, with justifications critiqued by Hui and Ruina [24] and Labossiere and Dunn [25].

For many combinations of materials, two unequal and real exponents are found, which are labeled such that $\lambda_{1}>\lambda_{2}$. This paper focuses on this case. The stress around the corner is a linear superposition of the two modes of singular fields:

$$
\sigma_{i j}(r, \theta)=\frac{k_{1}}{(2 \pi r)^{\lambda_{1}}} \Sigma_{i j}^{1}(\theta)+\frac{k_{2}}{(2 \pi r)^{\lambda_{2}}} \Sigma_{i j}^{2}(\theta) .
$$

The angular functions, $\Sigma_{i j}^{1}(\theta)$ and $\Sigma_{i j}^{2}(\theta)$, are normalized such that $\Sigma_{r \theta}^{1}(0)=\Sigma_{r \theta}^{2}(0)=1$, and their full expressions are listed in Appendix A. $k_{1}$ and $k_{2}$ are called the stress intensity factors, analogous to the stress intensity factors $K_{I}$ and $K_{I I}$ for a crack.

The singular stress field, Eq. (3), is only valid within an annulus, as highlighted in Fig. 1(b). Within the process zone, where $r<\Lambda$, there may be nonlinear behavior of materials and geometric perturbations. Material defects and interfacial flaws may also appear. On the other hand, an outer boundary for the validity of Eq. (3) also exists. Because at a length scale close to 
the chip thickness $h$, the external boundary conditions will distort the radial variance of stresses characterized by Eq. (3). Therefore as long as the process zone is significantly smaller than the macroscopic chip height, i.e. $\Lambda<<h$, the singular stress field governed by Eq. (3) will prevail within an annulus, known as the $k$-annulus. In the following discussion we will mainly focus on the singular stress field within the $k$-annulus.

In fracture mechanics, so long as the square-root singular fields prevail in an annulus, the stress intensities, $K_{I}$ and $K_{I I}$, are the only parameters that link external loadings to the material response inside the process zone. Consequently, the stress intensity factors can be used to formulate critical conditions for crack extension. In the same spirit, $k_{1}$ and $k_{2}$ may be used to formulate critical conditions to initiate failure. [24, 26, 27]

Equation (3) indicates that, in general, two singular modes, Mode 1 and Mode 2, govern the stress field in the $k$-annulus. For example, consider a homogeneous body with a sharp notch (Fig. 2). We use dash-dot-dash line to highlight the axis of symmetry of the body. Any external loading condition can be decomposed into a symmetric mode (Mode 1) as shown in Fig. 2(a) and an anti-symmetric mode (Mode 2) as shown in Fig. 2(b). Mode 1 corresponds to pure tension and Mode 2 represents pure shear with respect to the symmetric axis.

For a bimaterial corner, however, no such axis of symmetry exists, so that no simple geometric interpretation of the two singular modes is available. If we fix the opening angle $\varphi=90^{\circ}$ as shown in Fig. 1(b) but vary the elastic constants of the two materials, the singular exponents will change accordingly. Solving the eigenvalue problem, we plot $\lambda_{1}$ and $\lambda_{2}$ as functions of the Dundurs parameter, $\alpha$, in Fig. 3, with $\beta$ fixed to be zero. The plot shows that, while $\lambda_{1}$ is always close to $0.5, \lambda_{2}$ varies substantially with the elastic mismatch. When $\alpha \rightarrow-1$ i.e. $E_{c} / E_{s} \rightarrow 0, \lambda_{2}$ goes to zero. When $\alpha \rightarrow 1$, namely $E_{c} / E_{s} \rightarrow \infty, \lambda_{2}$ approaches 0.5 . 
Indicated in Fig. 3 are the singular components for two combinations of materials: Si/LTCC and Si/FR4.

When $\lambda_{1} \neq \lambda_{2}$, according to Eq, (3), $k_{1}$ and $k_{2}$ have different dimensions, (stress)(lengh) $)^{\lambda_{1}}$ and (stress)(lengh) ${ }^{\lambda_{2}}$, respectively. Hence the ratio of Mode 2 to Mode 1 stress can be written as $\left(k_{2} / k_{1}\right) r^{\lambda_{1}-\lambda_{2}}$, where $r$ can be an arbitrary length. [18] Labossiere et al. [28] have used this mode mixity in describing their experimental data. A similar approach was taken to describe the split singularities around a bimaterial interfacial crack tip. [29]

To quantify the mode mixity we fix the arbitrary length scale to be the process zone size $\Lambda$. Thus, a mode angle, $\psi$, is defined as

$$
\tan \psi=\frac{\mathrm{k}_{2}}{\mathrm{k}_{1}} \Lambda^{\lambda_{1}-\lambda_{2}}
$$

When the stress state at $r=\Lambda$ is pure Mode $1, \psi=0^{\circ}$; when it is pure Mode 2, $\psi=90^{\circ}$.

Mode 2 has often been neglected due to the fact that the Mode 2 stress field is always less singular than Mode 1, as indicated in Fig. 3. [6, 30, 31] Moreover, it is much simpler to use one stress intensity factor to implement a fracture initiation criterion at the bimaterial interface corner. However, whether Mode 2 is negligible or not depends on its relative significance compared to Mode 1 , which is captured by the mode angle. In the following, we will calculate the mode angles for chip-substrate systems with different substrate materials or geometries under various loading conditions. Our results provide insights into situations when Mode 2 can be significant.

\section{Calculation of stress intensity factors}

The two stress intensity factors $k_{1}$ and $k_{2}$ are determined by solving boundary-value

problems. As depicted in Fig. 4, we will solve boundary value problems under two loading 
conditions: stretching of the substrate and bending of the substrate. During processing, the substrate is also subject to a change in temperature, leading to stress concentration around chipsubstrate corners (Fig. 5). As shown in Appendix B, the change in temperature and the stretching stress give rise to the same stress intensity factors at the corner, provided the stretching stress is identified as

$$
p=\bar{E}_{s}\left[\left(1+v_{s}\right) \alpha_{s}-\left(1+v_{c}\right) \alpha_{c}\right] \Delta T
$$

where $\alpha_{c}$ and $\alpha_{\mathrm{s}}$ are the respective coefficients of thermal expansion, $\bar{E}_{s}=E_{s} /\left(1-v_{s}^{2}\right)$ is the plane strain modulus of the substrate and $\Delta T$ the change in temperature.

Let $P$ be the stretching force, and $M$ be the bending moment per unit thickness. Dimensional analysis dictates that the stress intensity factors, $k_{1}$ and $k_{2}$, relate to the loading parameters, $P$ and $M$, through

$$
\begin{aligned}
& \frac{k_{1}}{h^{\lambda_{1}}}=b_{11} \cdot \frac{P}{h}+b_{12} \cdot \frac{M}{h^{2}} \\
& \frac{k_{2}}{h^{\lambda_{2}}}=b_{21} \cdot \frac{P}{h}+b_{22} \cdot \frac{M}{h^{2}}
\end{aligned}
$$

Because the boundary-value problems are linear, the $b$ coefficients are independent of the loading parameters, but depend on material properties and various dimensionless ratios, including $E_{c} / E_{s}, H / h, L / h$, and $S / h$.

We solve the two boundary-value problems by using the commercial finite element method (FEM) code ABAQUS 6.7. Plane strain conditions are assumed. For each boundary value problem, the stress field along $\theta=0^{\circ}$ as a function of $r$, for example $\tau_{r \theta}(r, \theta=0)$, can be extracted from the FEM results. To make sure it falls in the $k$-annulus, we take $10^{-3} h<r<10^{-2} h$. 
According to Eq. (3) and the way we normalize the angular functions, the stress field along the interface is given by

$$
\tau_{r \theta}(r, \theta=0)=\frac{k_{1}}{(2 \pi r)^{\lambda_{1}}}+\frac{k_{2}}{(2 \pi r)^{\lambda_{2}}}
$$

The two stress intensity factors are determined by fitting the stress $\tau_{r \theta}(r, \theta=0)$ calculated from the FEM to Eq. (8). The boundary-value problem of stretching $(P=1, M=0)$ determines the coefficients $b_{11}$ and $b_{21}$. The boundary-value problem of bending $(P=0, M=1)$ determines the coefficients $b_{12}$ and $b_{22}$.

Once the $b$ coefficients are determined, Eqs. (6) and (7) can be used to calculate the stress intensity factors under any combined loads of stretching, bending and change in temperature. Substituting Eqs. (6) and (7) into Eq. (4), we find that the mode angle $\psi$ is given by

$$
\tan \psi=\frac{\mathrm{b}_{21}+\mathrm{b}_{22} \cdot \frac{\mathrm{M}}{\mathrm{Ph}}}{\mathrm{b}_{11}+\mathrm{b}_{12} \cdot \frac{\mathrm{M}}{\mathrm{Ph}}}\left(\frac{\Lambda}{\mathrm{h}}\right)^{\lambda_{1}-\lambda_{2}}
$$

This equation relates the mode angle to the loading parameters $P$ and $M$. We will use this method to investigate the effects of substrate material and chip-substrate geometry on the relative significance of the two singular modes in the following sections.

The process zone size is set to be $\Lambda / h=10^{-3}$ in all the following calculations. Since $0<\Lambda / h<<1$ and $\lambda_{1}-\lambda_{2}>0$, a larger value of $\Lambda / h$ yields a larger value of $(\Lambda / h)^{\lambda_{1}-\lambda_{2}}$, and hence a larger absolute value of $\psi$. However, so long as we keep a consistent choice of the process zone size, the trends discussed below are valid.

\section{Effect of the substrate material}


A simplified 2D chip-substrate structure is sketched in Fig. 1(a). The chip is of Si, and the substrate can be of either FR4 or LTCC. The Dundurs parameter for Si/FR4 and Si/LTCC systems are $\alpha \approx 0.70$ (large elastic mismatch) and $\alpha \approx 0.03$ (small elastic mismatch), respectively. The singular exponents of the two systems are also provided in Table 2 and highlighted in Fig. 3.

Figure 6 plots the mode angle $\psi$ as a function of the dimensionless ratio of the loading parameters, $M /(P h)$. Both structures, Si/FR4 and Si/LTCC, are considered. The general trends are noted below.

(a) Mode $2\left(\psi=90^{\circ}\right)$ dominates at a small absolute value of $M /(P h)$, where stretching prevails over bending.

(b) Mode $1\left(\psi=0^{\circ}\right)$ dominates at a large absolute value of $M /(P h)$, where bending prevails over stretching.

(c) Under most loading conditions, the larger elastic mismatch between the chip and the substrate, e.g. Si/FR4, has a larger Mode 2 component.

To understand these trends, we examine Eq. (9). Observe that $\psi=90^{\circ}$ when $M /(P h)=-b_{11} / b_{12}$. This is the point where $k_{1}=0$ according to Eq. (6). Referring to the $b$ coefficients listed in Table 2 we note that $-b_{11} / b_{12}=-0.1478$ for $\mathrm{Si} / \mathrm{FR} 4$ and $-b_{11} / b_{12}=-0.2384$ for $\mathrm{Si} / \mathrm{LTCC}$. Consequently, within a narrow band of $M /(P h)$ where pure stretching (or thermal loading) is dominant, Mode 2 can prevail over Mode 1.

When the external loading is bending dominant, i.e. $|\mathrm{M}|>>|\mathrm{Ph}|$, Eq. (9) shows that the mode angle $\psi$ approaches a constant 


$$
\psi=\tan ^{-1}\left[\frac{b_{22}}{b_{12}}\left(\frac{\Lambda}{h}\right)^{\lambda_{1}-\lambda_{2}}\right]
$$

which is calculated to be $1.49^{\circ}$ for Si/FR4 and $1.36^{\circ}$ for Si/LTCC. Therefore we conclude that when the chip-substrate is under pure bending or bending overwhelms stretching, the singular stress field is almost pure Mode 1.

The major difference between the two curves is the decay rate of the mode angle, from $90^{\circ}$ to almost $0^{\circ}$. If we take $|\psi|>45^{\circ}$ to be the criterion for Mode 2 dominance, substitute Eq. (9) in and solve the inequality we can determine the range of $M /(P h)$ for Mode 2 to be overwhelming:

$$
-\frac{b_{11}+\left(\frac{\Lambda}{h}\right)^{\lambda_{1}-\lambda_{2}} b_{21}}{\left(\frac{\Lambda}{h}\right)^{\lambda_{1}-\lambda_{2}} b_{22}+b_{12}}<\frac{M}{P h}<\frac{b_{11}-\left(\frac{\Lambda}{h}\right)^{\lambda_{1}-\lambda_{2}} b_{21}}{\left(\frac{\Lambda}{h}\right)^{\lambda_{1}-\lambda_{2}} b_{22}-b_{12}}
$$

Using the values of $\lambda$ and $b$ listed in Table 2, we find that when $-0.244<M /(P h)<-0.232$, $|\psi|>45^{\circ}$ for Si/LTCC. For Si/FR4, the band for $|\psi|>45^{\circ}$ widens to be $-0.210<M /(P h)<-0.082$. This observation indicates that the larger the elastic mismatch between chip and substrate, the bigger range of $M /(P h)$ for Mode 2 component to be significant.

In summary, Fig. 6 illustrates the relative contribution of the two singular modes at arbitrary combinations of $M /(P h)$ for two substrate materials, FR4 and LTCC. In both cases, when $M /(P h)$ approaches $-b_{11} / b_{12}$ from either side, the mode angle $\psi$ increases drastically until pure Mode 2 is reached. When $M /(P h)$ remains far from $-b_{11} / b_{12}$, the mode angle $\psi$ stays close to $0^{\circ}$ which means the singular stress field is Mode 1 dominant. A significant difference between two substrate materials lies in the decay rate of the mode angle: when the substrate material is 
FR4, i.e. when there is a large elastic mismatch between chip and substrate, $\psi$ decays slowly from $90^{\circ}$ to almost $0^{\circ}$. As a result, Mode 2 remains significant over a bigger range of $M /(P h)$.

\section{Geometric effects}

The geometry of the chip-substrate structure can also play an important role in determining the behavior of the singular stress field. We first vary the length of the chip, $L$, from $6 \mathrm{~mm}$ to $20 \mathrm{~mm}$, while keeping the other lengths fixed at $h=0.7 \mathrm{~mm}, H=1.4 \mathrm{~mm}$, and $S=40$ $\mathrm{mm}$. The $b$ coefficients for different lengths of the chip remain unchanged. This conclusion recovers a previous result [32]: the stress intensity factor reaches a plateau when $L / h$ is greater than 10. That is, when the length of the chip exceeds several times the thickness of the chip, the stress field near one edge of the chip can no longer be affected by the presence of the other edge. Therefore, further increase in $L$ cannot alter the singular stress field at the chip-substrate corner.

We study the effect of the size of the substrate by fixing $h=0.7 \mathrm{~mm}, L=10 \mathrm{~mm}$, and $H=$ $1.4 \mathrm{~mm}$, but varying $S$ from $15 \mathrm{~mm}$ to $60 \mathrm{~mm}$. The $b$ coefficients also remain constants when $S$ changes. That is, as long as the substrate is wide enough compared to the chip, regardless of how much further $S$ increases, the same boundary conditions on the two edges of the substrate will result in the same local stress field around the chip-substrate corner.

By contrast, the thickness of the substrate, $H$, significantly affects the behavior of the singular stress field. Table 3 and Fig. 7 shows the $b$ coefficients as functions of $H / h$. When $H / h$ is large, all the $b$ coefficients approach zero, indicating loadings on the edges of the substrate can no longer cause negligible singular stress around the chip-package corner. Individually, $b_{11}$ stays close to zero, indicating stretching makes little contribution to $k_{1}$. Stretching does contribute to $k_{2}$ because $b_{21}$ can be greater than 1 when $H / h$ is small; as $H / h$ increases, $b_{12}$ and $b_{22}$ decay to 
zero from much greater values than that of $b_{21}$, implying that the contribution from bending is much more sensitive to the change of the substrate thickness.

If we substitute the $b$ coefficients listed in Table 3 into Eq. (9), a plot of mode angle $\psi$ as a function of $M /(P h)$ can be obtained as shown in Fig. 8. As we discussed before, $\psi$ approaches $90^{\circ}$ as $M /(P h)$ goes to $-b_{11} / b_{12}$, which is also listed in Table 3 . Since $b_{11}$ remains close to zero but $b_{12}$ rises quickly as the substrate thickness decreases, the value of $-b_{11} / b_{12}$ shifts gradually from -0.4529 to almost 0 as the substrate gets thinner. When $M /(P h)$ is large, the value of $\psi$ increases slightly as the substrate gets thicker. The decay rate of the mode angle with respect to $M /(P h)$ is also affected by the substrate thickness: the thicker the substrate, the wider band of $M /(P h)$ where Mode 2 is significant.

\section{Concluding remarks}

In summary, we study singular stress field at corners in flip-chip packages, when the substrate is subject to a combination of bending and stretching. The singular stress field is a superposition of two modes, of dissimilar exponents, $\lambda_{1}>\lambda_{2}$. We compared the contributions of the two modes to the magnitude of the stress field at a length characteristic of the process zone size, $\Lambda$. We demonstrated that Mode 2 can contribute significantly when $M /(P h)$ approaches $-b_{11} / b_{12}$. When the loading is bending dominant, however, the Mode 2 component is always very small. At most loading conditions, Mode 2 component is more significant in a chipsubstrate system with larger elastic mismatch. While the lengths of the chip and the substrate have little effects on the singular stress field, the substrate-to-chip thickness ratio has a great influence. Thicker substrate shows larger range of $M /(P h)$ for Mode 2 to dominate over Mode 1. 


\section{Acknowledgment}

The work presented in this paper was supported by the National Science Foundation (NSF) under Grant CMS-0556169, and by the Materials Research Science and Engineering Center (MRSEC) at Harvard University. Juil Yoon thank the support from the Hansung University in the year of 2009.

\section{Appendix A. Stress components in polar coordinates}

The singular stress field Eq. (3) is solved by the methods outlined in Ref. [10, 5]. The

eigenfunctions $\Sigma_{i j}(\theta)$ associated with the eigenvalue $\lambda$ are expressed in polar coordinates $(r, \theta)$

as

$$
\begin{gathered}
\Sigma_{r r}(\theta)=-(\lambda-1)\{(\lambda-2)[A \sin (\lambda-2) \theta+B \cos (\lambda-2) \theta]+(\lambda+2)[C \sin \lambda \theta+D \cos \lambda \theta]\}, \\
\Sigma_{\theta \theta}(\theta)=(\lambda-1)(\lambda-2)[A \sin (\lambda-2) \theta+B \cos (\lambda-2) \theta+C \sin \lambda \theta+D \cos \lambda \theta], \\
\Sigma_{r \theta}(\theta)=(\lambda-1)\{(\lambda-2)[A \cos (\lambda-2) \theta-B \sin (\lambda-2) \theta]+\lambda[C \cos \lambda \theta-D \sin \lambda \theta]\}, \\
\Sigma_{z z}(\theta)=-4 v(\lambda-1)[C \sin \lambda \theta+D \cos \lambda \theta], \\
\Sigma_{r z}=\Sigma_{\theta z}=0 .
\end{gathered}
$$

The two eigenvalues $\lambda_{1}$ and $\lambda_{2}$ and the coefficients $A, B, C$, and $D$ in both the chip and the substrate are determined by solving the eigenvalue problem. The values of $A, B, C$ and $D$ are listed in Table A1.

\section{Appendix B. Representing thermal loading by uniform stretch on substrates}

As depicted in Fig. 5, FEM simulations of a 2D plane strain finite element model show that when the flip-chip package is subject to a temperature change, e.g. $\Delta T<0$, the singular 
stress fields at chip-substrate corners can be completely counteracted by a uniform uniaxial tensile stress $p$ exerting on the substrate. This appendix derives the relation between $p$ and $\Delta T$.

Firstly, assume the chip and the substrate are separated and become freestanding. Both the chip and the substrate are subject to thermal loading $\Delta T$ but the substrate has to be uniaxially stretched to cancel its strain mismatch with the chip. Then if the chip and the substrate are put together, no singular stress will be developed around the bimaterial interface corner, as shown in the middle configuration of Fig. 5. Referring to the coordinate shown in Fig. 5, plane strain condition requires $\varepsilon_{z}=0$.

In the chip

$$
\varepsilon_{z}^{(c)}=\frac{\sigma_{z}}{E_{c}}-v_{c} \frac{\sigma_{x}}{E_{c}}-v_{c} \frac{\sigma_{y}}{E_{c}}+\alpha_{c} \Delta T=0
$$

No traction is applied to the chip, hence $\sigma_{x}=\sigma_{y}=0$, solving Eq. (B1) gives

$$
\sigma_{z}=-E_{c} \alpha_{c} \Delta T
$$

Hence

$$
\varepsilon_{x}^{(c)}=\frac{\sigma_{x}}{E_{c}}-v_{c} \frac{\sigma_{y}}{E_{c}}-v_{c} \frac{\sigma_{z}}{E_{c}}+\alpha_{c} \Delta T=\left(1+v_{c}\right) \alpha_{c} \Delta T
$$

On the substrate, $\sigma_{x}=p, \sigma_{y}=0$

Plane strain condition requires

$$
\varepsilon_{z}^{(s)}=\frac{\sigma_{z}}{E_{s}}-v_{s} \frac{\sigma_{x}}{E_{s}}-v_{s} \frac{\sigma_{y}}{E_{s}}+\alpha_{s} \Delta T=0
$$

and $\sigma_{z}$ can be expressed in terms of $p$

$$
\sigma_{z}=v_{s} p-E_{s} \alpha_{s} \Delta T
$$

Therefore 


$$
\varepsilon_{x}^{(s)}=\frac{\sigma_{x}}{E_{s}}-v_{s} \frac{\sigma_{y}}{E_{s}}-v_{s} \frac{\sigma_{z}}{E_{s}}+\alpha_{s} \Delta T=\left(1-v_{s}^{2}\right) \frac{p}{E_{s}}+\left(1+v_{s}\right) \alpha_{s} \Delta T
$$

No mismatch between chip and substrate requires $\varepsilon_{x}^{(c)}=\varepsilon_{x}^{(s)}$. Solving the equation yields

$$
p=\frac{E_{s}}{\left(1-v_{s}^{2}\right)}\left[\left(1+v_{c}\right) \alpha_{c}-\left(1+v_{s}\right) \alpha_{s}\right] \Delta T
$$

If a 3D configuration is considered, an equivalent biaxial tensile stress $p_{3 \mathrm{D}}$ can be derived in a similar way:

$$
p_{3 D}=\frac{E_{s}}{\left(1-v_{s}\right)}\left(\alpha_{c}-\alpha_{s}\right) \Delta T
$$




\section{References}

[1] Atluri VP, Mahajan RV, Patel PR, Mallik D, Tang J, Wakharkar VS, Chrysler GM, Chiu CP, Choksi GN, Viswanath RS. Critical aspects of high-performance microprocessor packaging. MRS Bull. 2003;28(1):21-34.

[2] Reuss RH, Chalamala BR. Microelectronics packaging and integration. MRS Bull. 2003;28(1):11-15.

[3] Tummala RR, Raj PM, Atmur S, Bansal S, Banerji S, Liu FH, Bhattacharya S, Sundaram V, Shinotani KI, White G. Fundamental limits of organic packages and boards and the need for novel ceramic boards for next generation electronic packaging. J Electroceram. 2004;13(13):417-422.

[4] Frear DR, Thomas S. Emerging materials challenges in microelectronics packaging. MRS Bull. 2003;28(1):68-74.

[5] Madenci E, Shkarayev S, Mahajan R. Potential failure sites in a flip-chip package with and without underfill. J Electron Packag. 1998;120(4):336-341.

[6] Qian Z, Akisanya AR. An experimental investigation of failure initiation in bonded joints. Acta Mater. 1998;46(14):4895-4904.

[7] Chen L, Zhang Q, Wang GZ, Xie XM, Cheng ZN. The effects of underfill and its material models on thermomechanical behaviors of a flip chip package. IEEE Trans on Adv Packag. 2001;24(1):17-24.

[8] Fan XJ, Wang HB, Lim TB. Investigation of the underfill delamination and cracking in flip-chip modules under temperature cyclic loading. IEEE Trans Compon Packag Technol. 2001;24(1):84-91.

[9] Gu Y, Nakamura T, Chen WT, Cotterell B. Interfacial delamination near solder bumps and UBM in flip-chip packages. J Electron Packag. 2001;123(3):295-301.

[10] Choi WJ, Yeh ECC, Tu KN. Mean-time-to-failure study of flip chip solder joints on Cu/Ni(V)/Al thin-film under-bump-metallization. J Appl Phys. 2003;94(9):5665-5671.

[11] Mercado LL, Wieser H, Hauck T. Multichip package delamination and die fracture analysis. IEEE Trans on Adv Packag. 2003;26(2):152-159.

[12] Zhai CJ, Sidharth, Blish RC, Master RN. Investigation and minimization of underfill delamination in flip chip packages. IEEE Trans Device Mater Reliab. 2004;4(1):86-91.

[13] Kwon WS, Yim MJ, Paik KW, Ham SJ, Lee SB. Thermal cycling reliability and delamination of anisotropic conductive adhesives flip chip on organic substrates with emphasis on the thermal deformation. J Electron Packag. 2005;127(2):86-90.

[14] Wang GT, Merrill C, Zhao JH, Groothuis SK, Ho PS. Packaging effects on reliability of Cu/Low-k interconnects. IEEE Trans Device Mater Reliab. 2003;3(4):119-128.

[15] Dundurs J. Discussion of a paper by D. B. Bogy. J Appl Mech. 1969;36:650-652.

[16] Dempsey JP, Sinclair GB. Stress singularities in the plane elasticity of the composite wedge. J Elast. 1979;9(4):373-391.

[17] Dempsey JP, Sinclair GB. On the singular behavior at the vertex of a bi-material wedge. J Elast. 1981;11(3):317-327.

[18] Liu XH, Suo Z, Ma Q. Split singularities: stress field near the edge of a silicon die on a polymer substrate. Acta Mater. 1998;47(1):67-76.

[19] Im S, Kim KS. An application of two-state M-integral for computing the intensity of the singular tip field for a generic wedge. J Mech Phys Solids. 2000;48(1):129-151. 
[20] Williams ML. Stress singularities resulting from various boundary conditions in angular corners of plates in extension. J Appl Mech. 1952;19(4):526-528

[21] Bogy DB. 2 edge-bonded elastic wedges of different materials and wedge angles under surface tractions. J Appl Mech. 1971;38(2):377.

[22] Hein VL, Erdogan F. Stress singularities in a 2-material wedge. Int J Fract Mech. 1971;7(3):317.

[23] Zhang Z, Suo Z. Split singularities and the competition between crack penetration and debond at a bimaterial interface. Int J Solids Struct. 2007;44:4559-4573.

[24] Hui CY, Ruina A. Why k-high-order singularities and small-scale yielding. Int J Fract. 1995;72(2):97-102.

[25] Labossiere PEW, Dunn ML. Fracture initiation at three-dimensional bimaterial interface corners. J Mech Phys Solids. 2001;49(3):609-634.

[26] Reedy ED. Connection between interface corner and interfacial fracture analyses of an adhesively-bonded butt joint. Int J Solids Struct. 2000;37(17):2429-2442.

[27] Dunn ML, Hui CY, Labossiere PEW, Lin YY. Small scale geometric and material features at geometric discontinuities and their role in fracture analysis. Int J Fract. 2001;110(2):101-121.

[28] Labossiere PEW, Dunn ML, Cunningham SJ. Application of bimaterial interface corner failure mechanics to silicon/glass anodic bonds. J Mech Phys Solids. 2002;50(3):405-433.

[29] Rice JR. Elastic fracture-mechanics concepts for interfacial cracks. J Appl Mech-Trans ASME. 1988;55(1):98-103.

[30] Dunn ML, Cunningham SJ, Labossiere PEW. Initiation toughness of silicon/glass anodic bonds. Acta Mater. 2000;48(3):735-744.

[31] Reedy ED, Guess TR. Nucleation and propagation of an edge crack in a uniformly cooled epoxy/glass bimaterial. Int J Solids Struct. 2002;39(2):325-340.

[32] Zhang Z, Yoon J, Suo Z. Method to analyze dislocation injection from sharp features in strained silicon structures. Appl Phys Lett. 2006;89:261912.

[33] Shin KC, Kim WS, Lee JJ. Application of stress intensity to design of

anisotropic/isotropic bi-materials with a wedge. Int J Solids Struct. 2007;44(24):7748-7766. 
Table 1 Material properties for chip and substrates used in calculation

\begin{tabular}{c|c|c|c}
\hline & $E(\mathrm{GPa})$ & $v$ & $\mathrm{CET}(\mathrm{ppm} / \mathrm{C})$ \\
\hline Silicon & 130 & 0.28 & 3.3 \\
FR4 & 23 & 0.3 & 15 \\
LTCC & 120 & 0.3 & 5.8 \\
\hline
\end{tabular}

Table 2 Dunders parameters, singular exponents and $b$ coefficients for two chip-package systems with fixed geometry: $h=0.7 \mathrm{~mm}, L=10 \mathrm{~mm}, H=1.4 \mathrm{~mm}$, and $S=20 \mathrm{~mm}$, as shown in Fig. 1(a).

\begin{tabular}{c|c|c|c|c|cccc}
\hline & $\alpha$ & $\beta$ & $\lambda_{1}$ & $\lambda_{2}$ & $b_{11}$ & $b_{12}$ & $b_{21}$ & $b_{22}$ \\
\hline Si/FR4 & 0.6961 & 0.1959 & 0.4990 & 0.3178 & 0.1117 & 0.7560 & 0.1785 & 0.0689 \\
Si/LTCC & 0.0337 & 0 & 0.4571 & 0.0996 & 0.1049 & 0.4401 & 0.0617 & 0.1238 \\
\hline
\end{tabular}

Table $3 b$ coefficients for Si/FR4 chip-package system with various substrate thicknesses, where $h=0.7 \mathrm{~mm}, L=10 \mathrm{~mm}$, and $S=20 \mathrm{~mm}$ are fixed

\begin{tabular}{c|cccc|c}
\hline \hline$H / h$ & $b_{11}$ & $b_{12}$ & $b_{21}$ & $b_{22}$ & $-b_{11} b_{12}$ \\
\hline $1 / 3$ & -0.0193 & 13.6450 & 1.2091 & -4.2503 & 0.0014 \\
$1 / 2$ & 0.0077 & 6.9809 & 0.8595 & -1.2846 & -0.0011 \\
1 & 0.0710 & 2.3352 & 0.4234 & -0.1236 & -0.0304 \\
2 & 0.1117 & 0.7560 & 0.1785 & 0.0689 & -0.1478 \\
4 & 0.1085 & 0.2396 & 0.0784 & 0.0524 & -0.4529 \\
\hline
\end{tabular}

Table A1 Coefficients appearing in eigenfunctions given by Eqs. (A.1) to (A.4)

\begin{tabular}{c|c|c|c|c|c|c|c|c}
\hline \hline & \multicolumn{4}{|c|}{ Mode 1 } & \multicolumn{4}{c}{ Mode 2 } \\
\cline { 2 - 9 } & $A_{1}$ & $B_{1}$ & $C_{1}$ & $D_{1}$ & $A_{2}$ & $B_{2}$ & $C_{2}$ & $D_{2}$ \\
\hline $\mathrm{Si}$ & 0.5752 & -0.8429 & -2.2700 & 1.9937 & 0.9989 & 0.2841 & -0.9955 & 0.8666 \\
$\mathrm{FR} 4$ & 0.0010 & -1.7404 & -4.6075 & 0.7720 & 0.6337 & -0.6262 & -1.2579 & -0.3421 \\
\hline $\mathrm{Si}$ & 0.9937 & 0.3675 & -0.6756 & 1.8257 & 0.9889 & 0.3764 & -0.6915 & 1.8169 \\
LTCC & 0.1349 & -1.9634 & -8.5779 & -0.5894 & 0.1623 & -1.8439 & -8.0551 & -0.7089 \\
\hline
\end{tabular}




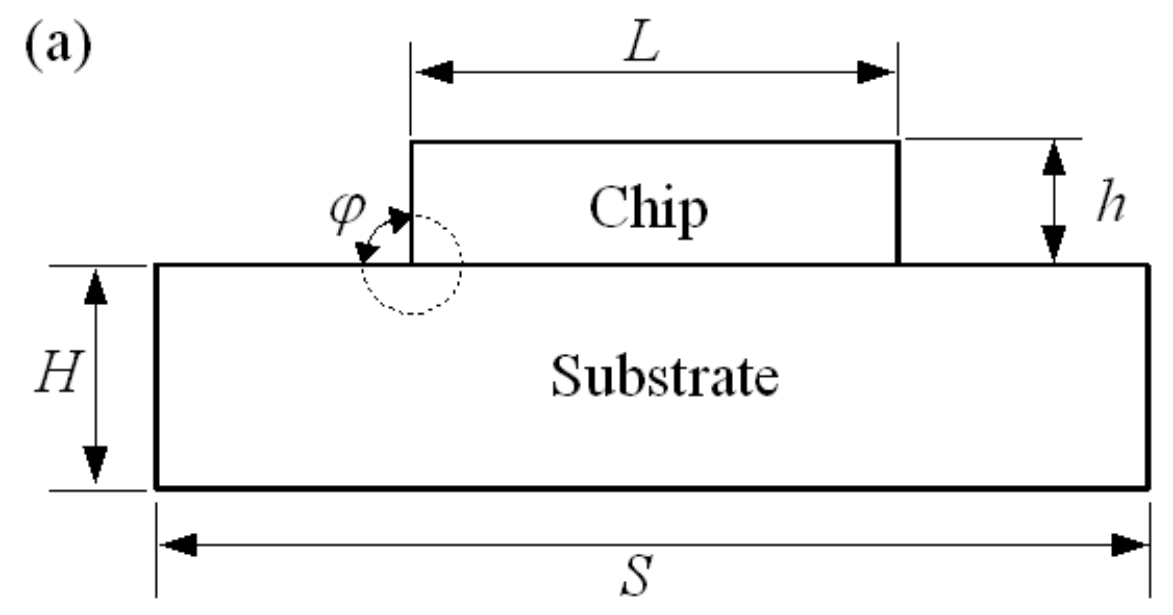

(b)

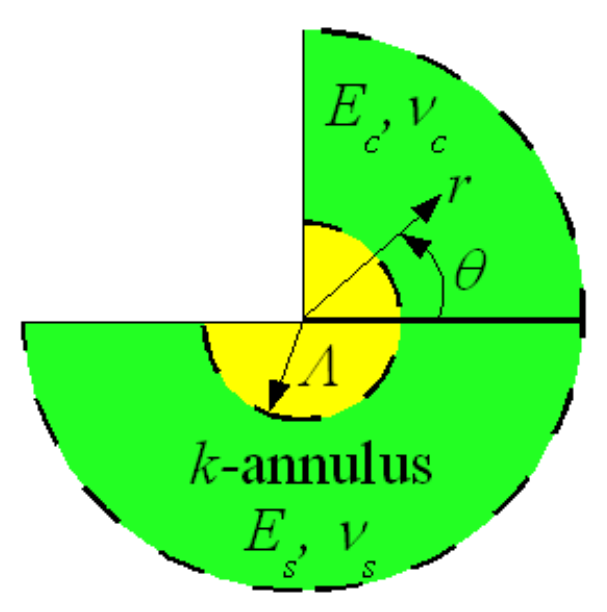

Figure 1 (a) Simplified 2D chip-substrate model with materials listed in Table 1. Plane strain conditions are assumed. (b) Blown up chip-substrate interface corner highlighted in Fig. 1(a) with process zone of radius $\Lambda$ and the $k$-annulus. A system of polar coordinates $(r, \theta)$ is defined. 


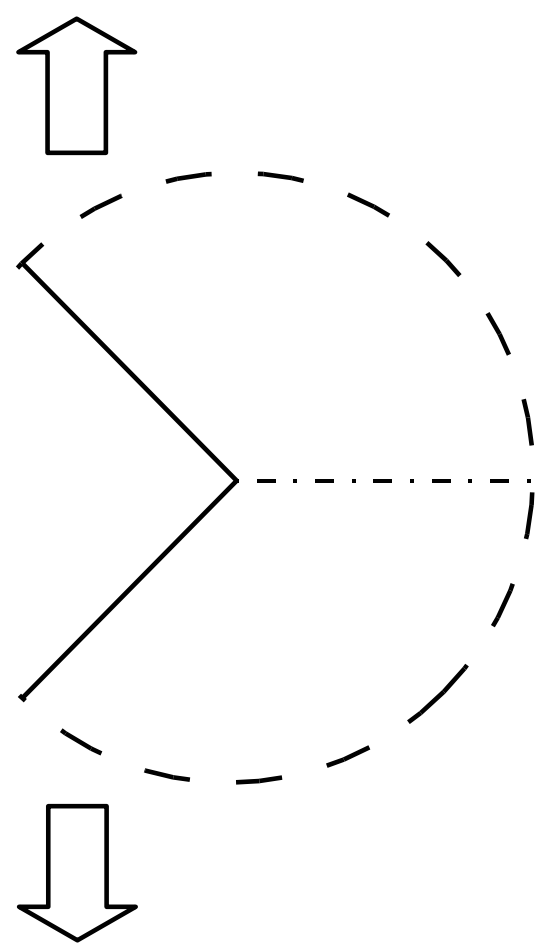

(a) Mode 1

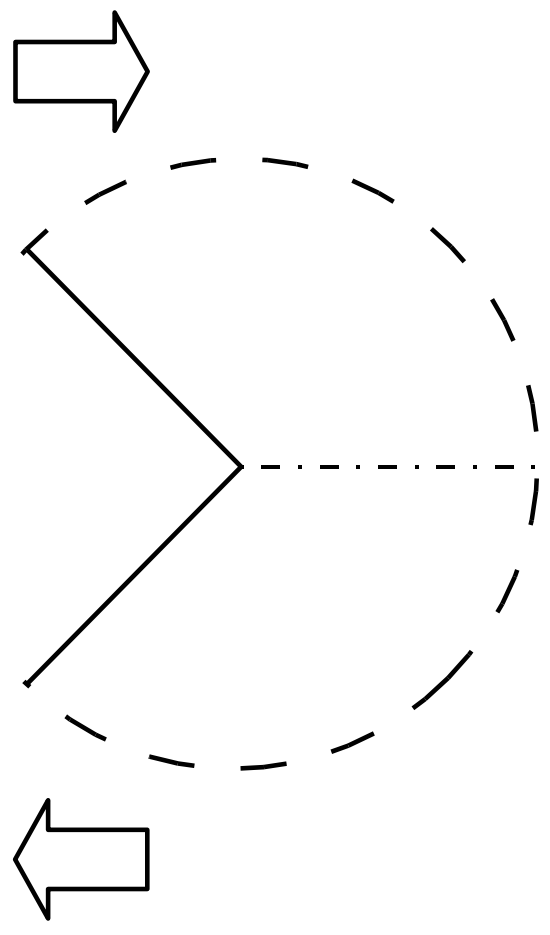

(b) Mode 2

Figure 2 Geometric interpretations of two modes in a homogeneous body with a sharp notch. The symmetric axis of the structure is highlighted by the dot-dash-dot line. Any external loading can be decomposed into (a) the symmetric mode (Mode 1) and (b) the anti-symmetric mode (Mode 2). 


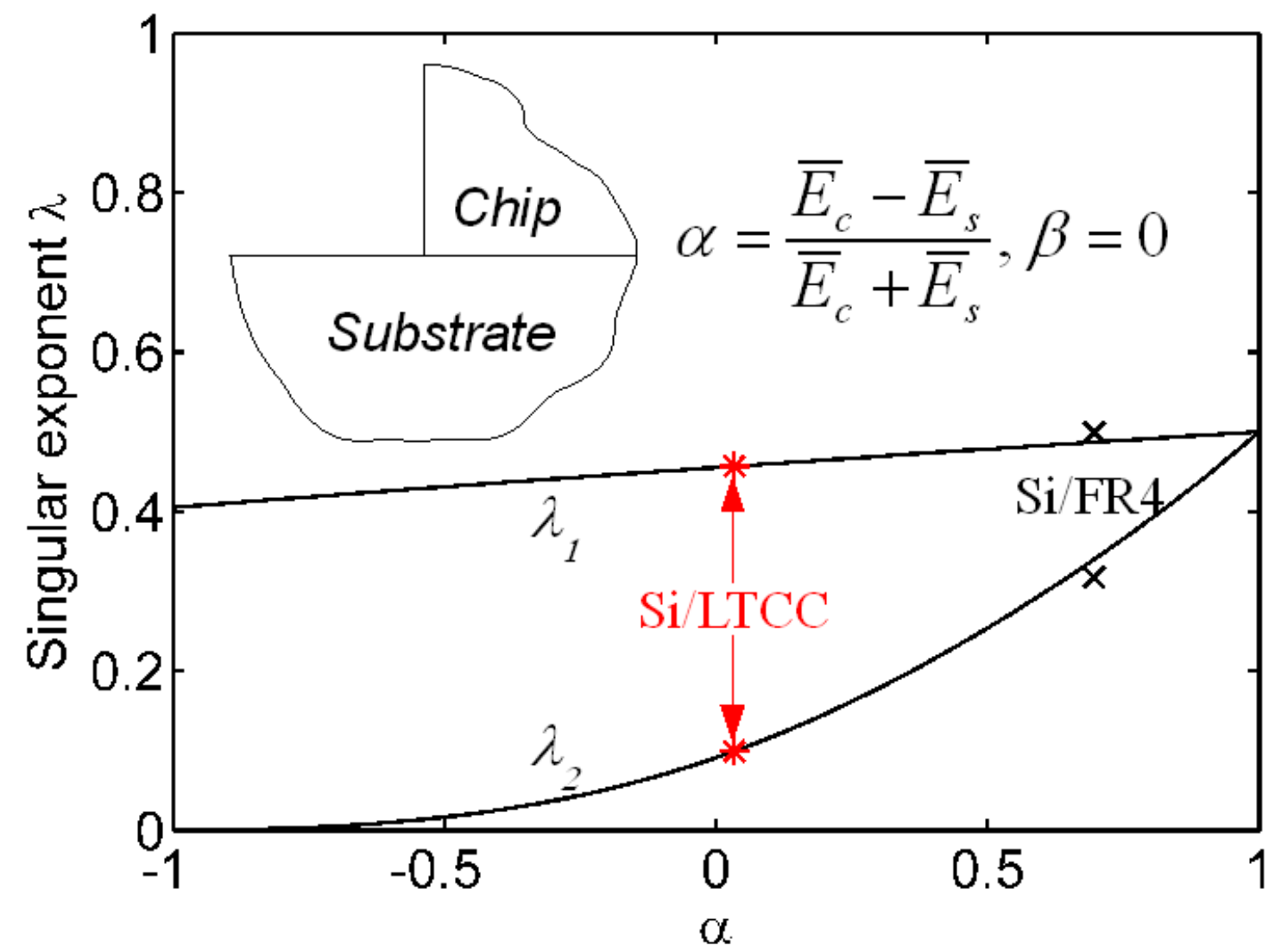

Figure 3 Split singularities at orthogonal interface corner as functions of elastic mismatch, denoted by Dundurs parameter $\alpha$, with $\beta$ fixed to be zero. Stars highlight the singular exponents when Chip $=$ Si and Substrate $=$ LTCC. Crosses show the singular exponents for Chip $=$ Si and Substrate $=$ FR4. Since $\beta$ is not exactly zero in this case, the points are slightly off the curves. 

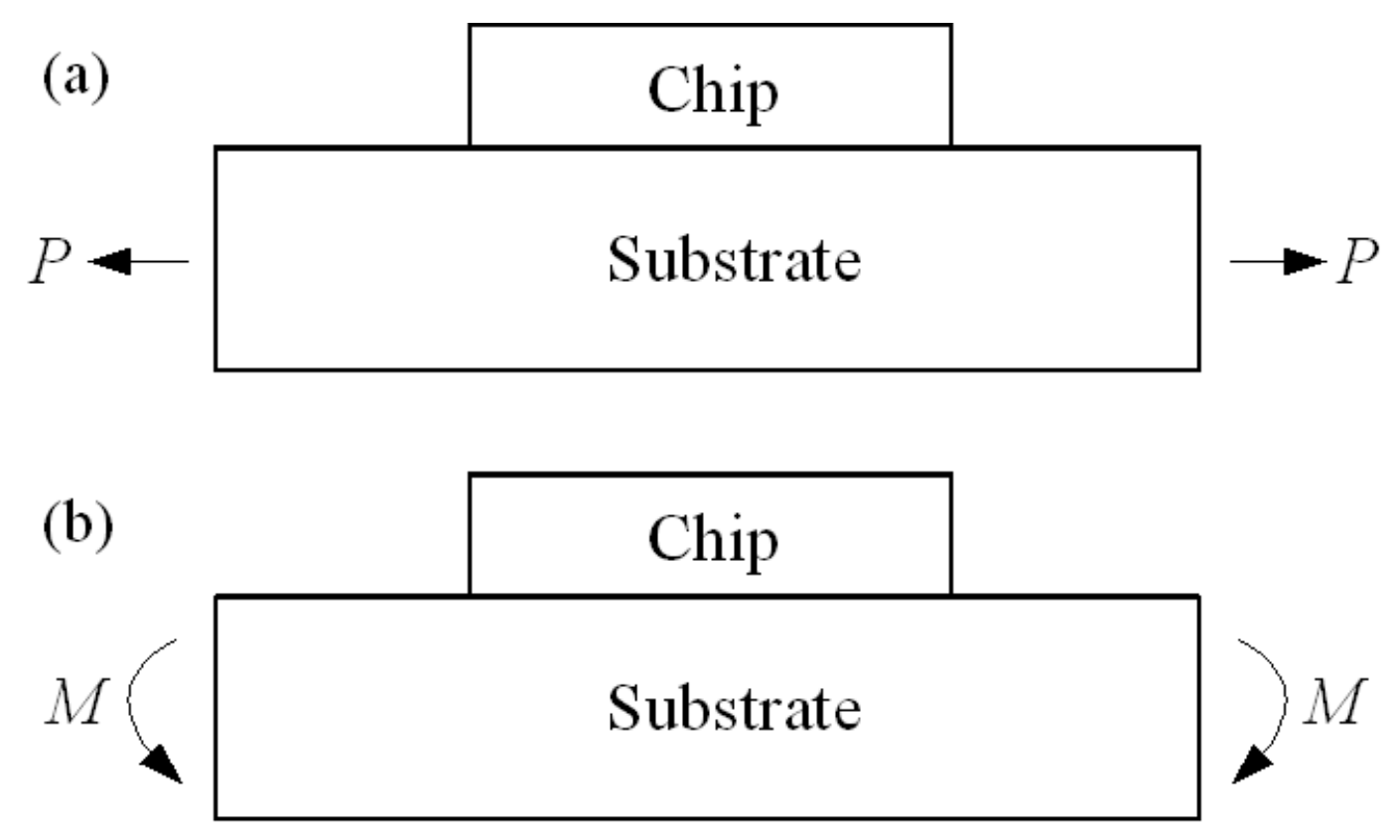

Figure 4 Two linearly independent loading conditions: (a) stretching force $P$ applied on the substrate; (b) bending moment $M$ applied on the substrate. 

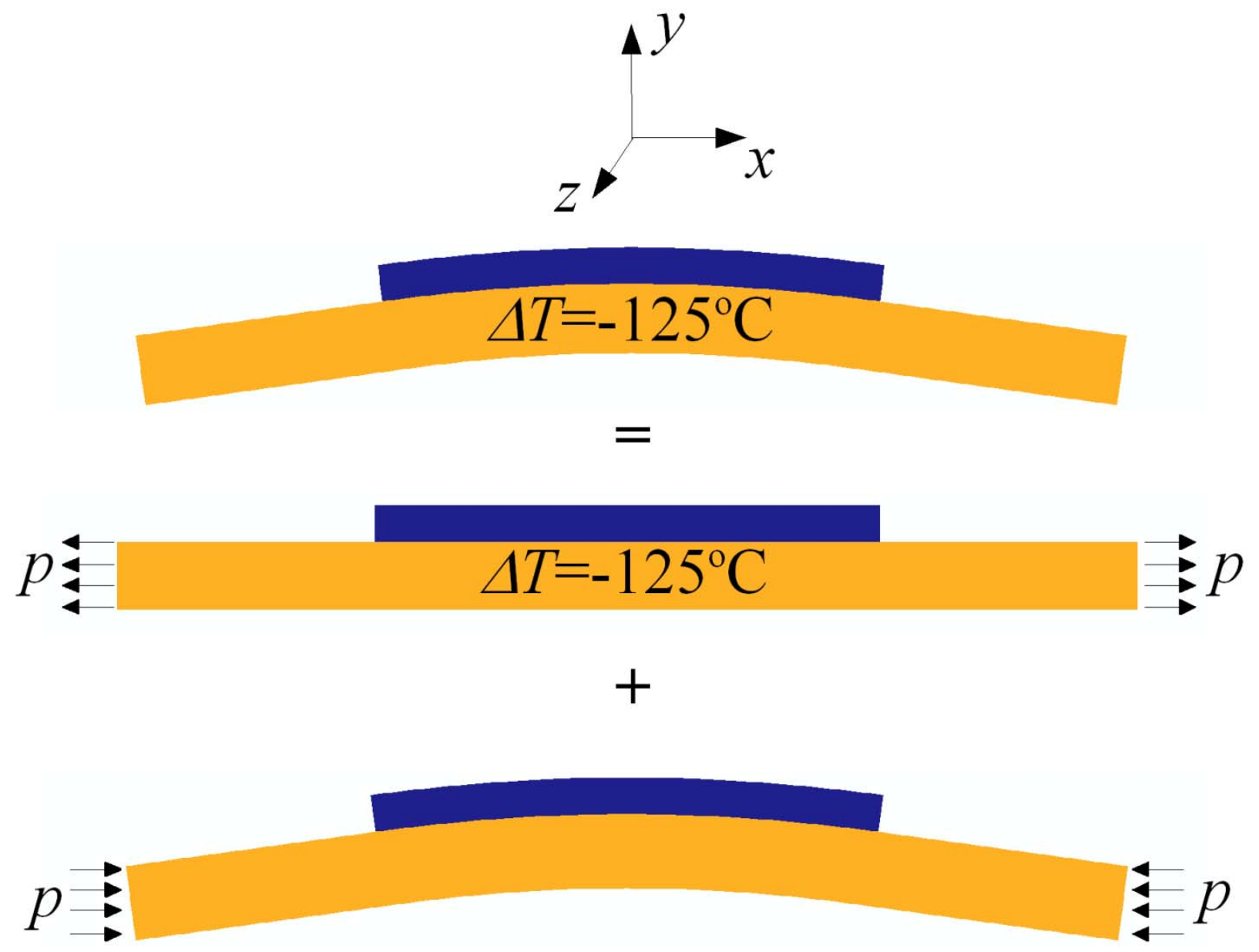

Figure 5 Equivalent singular stress fields around chip-substrate corners can be generated by either thermal loading $\Delta T$ on the chip-substrate system or uniform uniaxial tension $p=\bar{E}_{s} \Delta T\left[\left(1+v_{c}\right) \alpha_{c}-\left(1+v_{s}\right) \alpha_{s}\right]$ on the substrate, as derived in Appendix B. All pictures are direct outputs from ABAQUS results. 


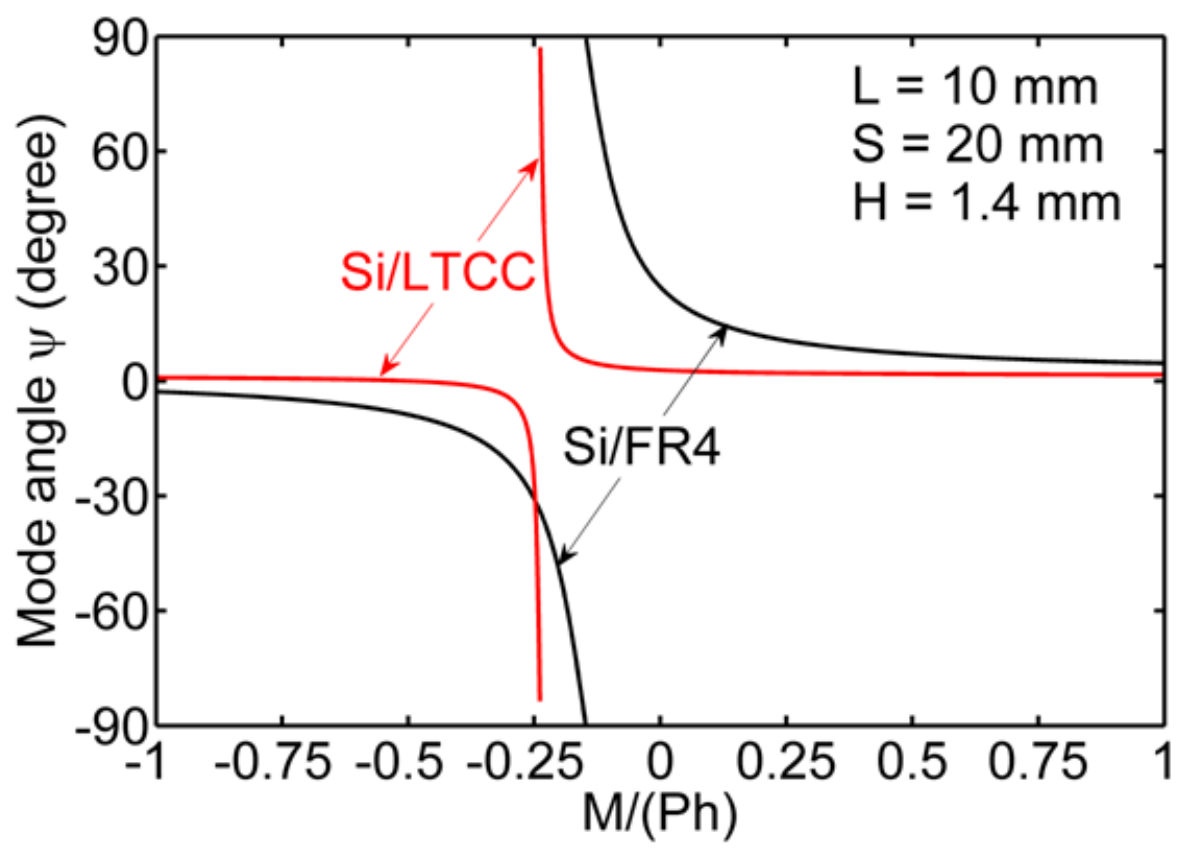

Figure 6 Mode angle as a function of the proportion of bending to stretching for two different substrate materials: FR4 and LTCC. 

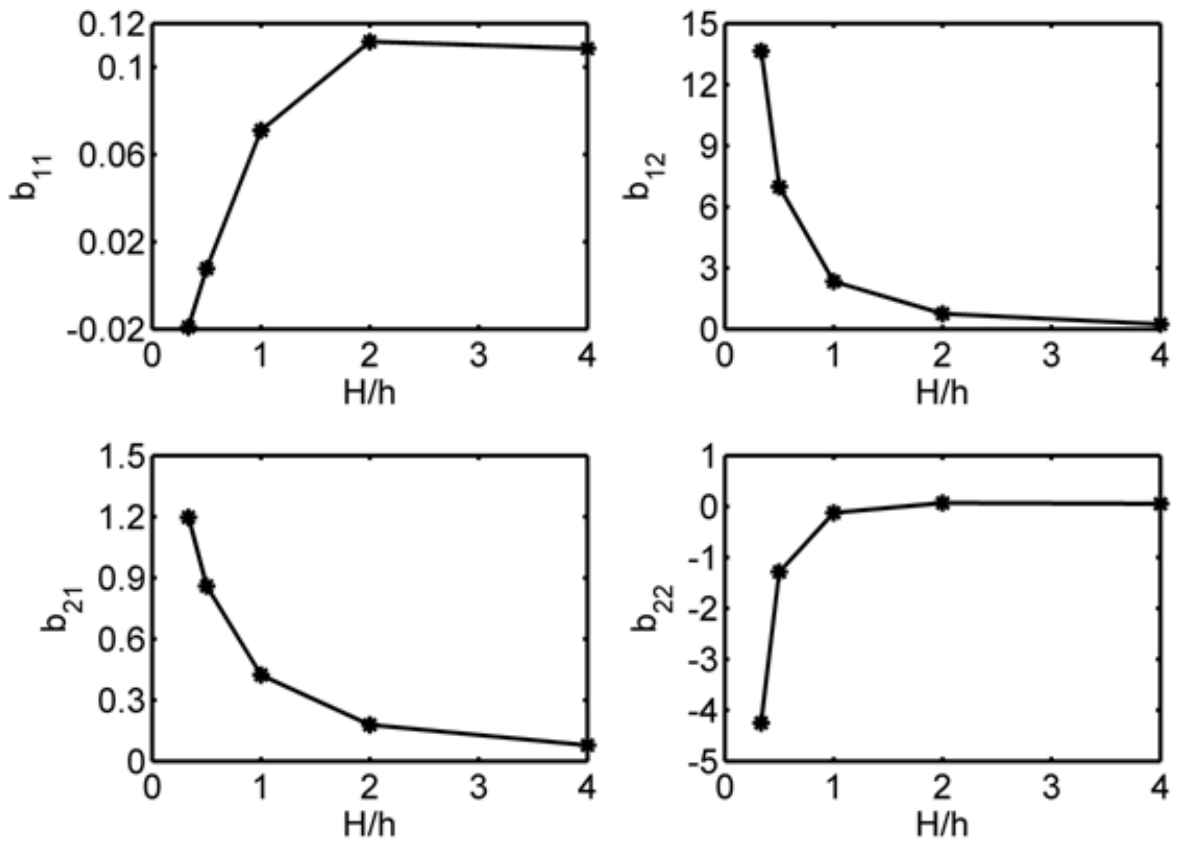

Figure $7 b$ coefficients as functions of substrate-to-chip thickness ratio with fixed sizes of chip and substrate, $L=10 \mathrm{~mm}$ and $S=20 \mathrm{~mm}$ respectively. 


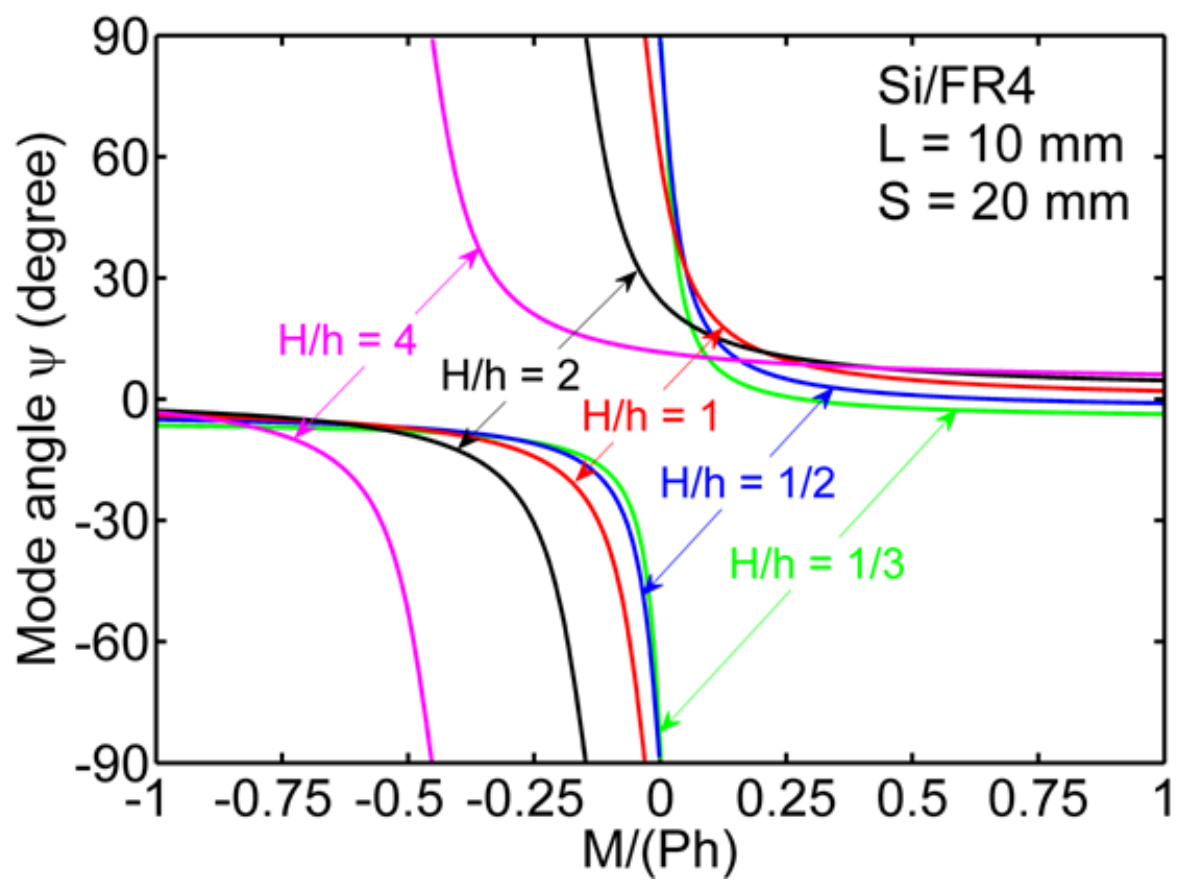

Figure 8 Mode angle as a function of the proportion of bending to stretching for Si/FR4 chipsubstrate system with various substrate-to-chip thickness ratios. 